Cahiers $d u$ MONDE RUSSE

\section{Cahiers du monde russe}

Russie - Empire russe - Union soviétique et États indépendants

$56 / 4 \mid 2015$

Médiateurs d'empire en Asie centrale (1820-1928)

\title{
Benjamin Tromly, Making the Soviet Intelligentsia, Universities and Intellectual Life under Stalin and Khrushchev
}

Jutta SCHERRER

\section{(2) OpenEdition}

\section{Electronic version}

URL: http://journals.openedition.org/monderusse/8297

DOI: $10.4000 /$ monderusse. 8297

ISSN: $1777-5388$

Publisher

Éditions de l'EHESS

\section{Printed version}

Date of publication: 1 October 2015

Number of pages: $909-911$

ISBN: 978-2-7132-2507-9

ISSN: $1252-6576$

\section{Electronic reference}

Jutta SCHERRER, «Benjamin Tromly, Making the Soviet Intelligentsia, Universities and Intellectual Life under Stalin and Khrushchev », Cahiers du monde russe [Online], 56/4 | 2015, Online since 01 October 2015, Connection on 24 September 2020. URL : http://journals.openedition.org/monderusse/8297 ; DOI : https://doi.org/10.4000/monderusse.8297 


\section{Benjamin Tromly, Making the Soviet Intelligentsia, Universities and Intellectual Life under Stalin and Khrushchev}

Jutta SCHERRER

\section{REFERENCES}

Benjamin TROMLY, Making the Soviet Intelligentsia, Universities and Intellectual Life under Stalin and Khrushchev, Cambridge : Cambridge University Press, 2014, 295 p.

1 Pour Benjamin Tromly, professeur associé d'histoire européenne moderne à l'université américaine Puget Sound, la véritable formation, "the making», de l'intelligentsia soviétique a eu lieu dans les années d'après-guerre, au commencement de la guerre froide, quand l'Union soviétique a eu besoin de nouvelles structures et d'une industrie militaire appuyées sur l'avancement de diverses sciences.

2 Tromly pense ainsi pouvoir ignorer le bagage historique de l'ancienne intelligentsia russe des années vingt et trente, son émigration ou ses tentatives d'adaptation au régime soviétique, sa décimation sous la Grande Terreur et sa déportation au Goulag, mais aussi la proclamation de la nouvelle "intelligentsia soviétique ", censée constituer, selon les mots de Stalin, le troisième pilier de la société soviétique à côté des ouvriers et des paysans.

3 Pour Tromly l'intelligentsia n'est pas un groupe social unifié, mais un "social construct ", une "constellation of ideas about the place of the holders of learning in society» (p. 245). Surtout après la guerre, argumente Tromly, l'enseignement supérieur, et en particulier celui de la physique et des sciences naturelles, fut, en URSS, un moyen puissant d'avancement social et économique. 
4 Le livre de Tromly se divise en trois parties : « Universités et société soviétique après la guerre »; «Émergence de l'intelligentsia stalinienne, 1948-1956»; «Rêves révolutionnaires et divisions de l'intelligentsia, 1957-1964 ».

Le premier chapitre est un portrait positif, presque idyllique du milieu universitaire dans les premières années d'après-guerre. Il met particulièrement en relief le besoin des institutions soviétiques d'une intelligentsia professionnelle pour restructurer le pays dévasté par la guerre, la soif de savoir de la jeunesse après la guerre, ou encore des vétérans (jouissant de conditions d'admission particulières aux universités), l'impact de professeurs appartenant encore à l'intelligentsia prérévolutionnaire qui transmettaient ses anciennes vertus aux étudiants : tels Gudzij, professeur de littérature, ou l'historien Zajončkovskij. Le rôle du Komsomol et des unions d'étudiants, la lourdeur du "party-state» et le service de sécurité à l'intérieur des universités ainsi que la politique d'admission aux universités liée à l'« inévitable » corruption sont traités dans le deuxième chapitre.

6 La répercussion de la politique russo-centrée, patriotique et antisémite de Stalin et de sa campagne contre le cosmopolitisme sur la vie universitaire est abordée dans le troisième chapitre qui introduit la deuxième partie du livre intitulé « The emergence of Stalin's intelligentsia 1948-1956». Ce n'est que dans ce contexte que l'auteur revient sur le russo-centrisme chauvin déjà affiché par Stalin dans les années trente. Les dénonciations de professeurs et, à un moindre degré, aussi d'étudiants, reflètent un État qui se méfie de ses élites intellectuelles.

7 Le quatrième chapitre traite les "guerres scientifiques " dans les universités pendant les dernières années de Stalin. Les contributions à la linguistique de Stalin en personne, l'impact de Lysenko sur la génétique amènent, pour Tromly, des transformations importantes de l'identité de l'intelligentsia dans le corps des étudiants et de jeunes enseignants. Le débat sur la physique atomique, bien qu'il ait davantage concerné l'Académie des sciences, a ainsi entraîné à l'université de Moscou une réorganisation du département de physique en fonction des protestations des étudiants.

Le cinquième chapitre traite de la déstalinisation qui fut accompagnée par une agitation dans les universités, que Tromly explique comme "le produit d'une interaction entre enseignement, identité et crise du poststalinisme» (p. 131). Tromly décrit ici le vide idéologique laissé par le rapport de Hruščev en 1956, vide que les étudiants ont essayé de remplir. Comme ailleurs en Union soviétique, les universités étaient caractérisées aussi bien par leur soutien à la déstalinisation de Hruščev que par l'opposition à cette tentative. Le sous-chapitre sur le dégel, est excellent par sa description de l'ambivalence de cette libération idéologique, en expliquant l'« activisme culturel » des étudiants comme une réponse aux points faibles du système soviétique qui allait souvent de pair de la part des étudiants avec l'option d'une vision marxiste du socialisme démocratique.

9 Les chapitres six à huit (la troisième partie de l'ouvrage) couvrent la période 1957-1964. Le chapitre six décrit la critique de Hruščev de l'élitisme de l'intelligentsia, devenue pour lui une « self-producing caste » qui, grâce à ses privilèges, avait perdu la volonté de travailler pour la société. L'obligation du «travail dans la production» l'expérience du travail pratique - introduite en 1958 par Hruščev, est liée dès lors à l'admission aux universités, les étudiants ou la future intelligentsia étant considérés comme une classe de service et non comme une élite. C'est dans la même perspective que Hruščev mobilise les étudiants à participer à cultiver des «terres vierges ». Les 
réactions des étudiants, analysées par Tromly, jettent un regard neuf et révélateur sur les réformes de Hruščev. Le chapitre sept sur le "dégel des étudiants" ("student Thaw ») montre comment celui-ci portait l'empreinte du milieu de l'intelligentsia dont les étudiants, aux yeux de Tromly, faisaient partie. L'intelligentsia agit ici, grâce au dégel, comme Kulturträger. Le dégel a aussi influencé, comme le décrit Tromly au chapitre huit, des mouvements nationaux parmi les étudiants et en particulier dans les universités ukrainiennes.

10 L'ouvrage de Tromly se fonde sur une connaissance solide et approfondie de son sujet et représente une analyse et une réflexion tout à fait nouvelles sur l'époque traitée. L'auteur a consulté des archives à Moscou et à Kiev et a mené une quarantaine d'entretiens auprès d'anciens étudiants des universités de Moscou, Kiev et Saratov qui sont au centre de sa recherche. L'ouvrage, très bien rédigé, fournit beaucoup d'informations importantes et jusqu'ici inconnues sur la vie à l'intérieur de ces trois universités (avec quelques aperçus sur d'autres institutions de l'enseignement supérieur), ainsi que sur le rapport entre le milieu universitaire et l'État-parti dont le Komsomol. Dans tous les chapitres, l'auteur renvoie très utilement à l'état de la recherche historique actuelle, ce qui met non seulement en valeur sa propre argumentation sur le sujet, mais permet aussi au lecteur de comparer ses thèses avec les interprétations d'autres spécialistes. Les analyses du dégel et de ses répercussions sur les mouvements nationalistes dans les milieux universitaires en particulier ouvriront certainement une nouvelle perspective pour la recherche future de cette période.

11 Les différents chapitres sont d'importance inégale et leur contenu ainsi que leur chronologie se recoupent parfois, entrainant ainsi des répétitions qui auraient pu être évitées. Davantage de matériaux statistiques auraient pu renforcer encore l'argumentation de l'auteur sur les origines et la mobilité sociale du corps étudiant. De même, des informations sur le milieu des professeurs auraient également mérité d'être apportées.

On peut féliciter Tromly de ne pas avoir cédé à la tentation de mythifier l'ancienne intelligentsia et d'avoir évité de longues discussions sur l'emploi de ce terme, connu dans la littérature secondaire. On peut cependant contester sa volonté de situer le «making of the Soviet intelligentsia » exclusivement dans les années d'après-guerre, car ce processus avait commencé bien avant la fin du règne de Stalin. "Making a post-war intelligentsia» aurait peut-être mieux correspondu au contenu de son ouvrage ou encore le titre de sa thèse (soutenue en 2007 à l'université de Harvard) «Re-imagining the Soviet Intelligentsia: Student Politics and University Life 1948-1964».

13 Malgré ces remarques critiques, je voudrais souligner ici que la recherche de Benjamin Tromly présente une contribution innovante, instructive et très importante à notre connaissance de la vie universitaire soviétique entre la fin de la Seconde Guerre mondiale et l'ère Hruščev.

Enfin, la bibliographie russe et anglo-américaine remarquablement riche utilisée par l'auteur témoigne du grand sérieux de sa recherche. Au-delà de sa personne, il est permis de regretter qu'il soit aujourd'hui possible à un excellent spécialiste américain de l'histoire de l'Union soviétique d'ignorer presque totalement (hormis un article de Bella Ostromoukhova et un livre de Dietrich Beyrau) l'historiographie française ou allemande non moins riche en ce domaine. 


\section{AUTHORS}

\section{JUTTA SCHERRER}

EHESS, Paris 